\title{
Actinide-Specific Interfacial Chemistry of Monolayer Coated Mesoporous Ceramics
}

(Project Number: 65370)

\section{Principal Investigator}

Glen E. Fryxell

Pacific Northwest National Laboratory

P.O. Box 999, MSIN K2-44

Richland, WA 99352

(509) 375-3856 (phone)

(509) 375-2186 (fax)

glen.fryxell@pnl.gov

\section{Co-Investigators}

Tom S. Zemanian

Pacific Northwest National Laboratory

P.O. Box 999, MSIN P7-07

Richland, WA 99352

(509) 373-0344 (phone)

(509) 376-3002 (fax)

ts.zemanian@pnl.gov

Ken M. Kemner

Argonne National Laboratory

Argonne, IL 60439

(630) 252-1163 (phone)

Ken_Kemner@anl.gov

Ken Raymond

Department of Chemistry

University of California at Berkeley

Berkeley, CA 94720-1760

(510) 642-7219 (phone)

Raymond@socrates.berkeley.edu 


\section{Specific DOE Problem}

This research addresses selective separation, isolation, or sequestration of actinide species from complex waste mixtures for stabilization, characterization, quantification, and permanent disposition.

\section{Research Objective}

The objective of this program is to design, synthesize, and evaluate high-efficiency, highcapacity sorbent materials capable of selectively sequestering actinides from complex aqueous mixtures. One of the central goals of this project is to understand the fundamental interfacial science required to develop novel mesoporous materials coated with organized monolayers of rationally designed ligands, custom-tailored for binding specific actinide cations. This capability addresses waste management by separation of actinides, a central concern of high-level waste (HLW) and nuclear materials management at several DOE sites.

\section{Research Progress and Implications}

This report summarizes work after 1.5 years of a three-year project. Selective isolation of actinides from a complex mixture is anticipated to increase assay accuracy, speed up analysis turnaround time, reduce waste volume (and cost), and enhance decontamination efficiency and safety.

\section{Novel Silane Synthesis}

Extending our studies from last year, we continued to prepare and evaluate new interfacial ligand fields for our self-assembled monolayers on mesoporous supports (SAMMS) sorbent materials. An important class of ligands that have been exploited in actinide solution extraction methods are the carbamoylphosphine oxide (CMPO) ligands. We incorporated several variations of the fundamental CMPO structure into our monolayers (e.g., Ac-Phos, Prop-Phos, etc. $)^{(a)}$. We have also incorporated some of Professor Raymond's plutonium-specific ligands into the SAMMS materials, most notably the hydroxypyridinone (HOPO) and catechol ligands developed and synthesized by the Raymond Group.

\section{Supercritical Fluid (SCF) Methods}

In Year 1, we developed powerful new synthetic methods for the deposition of selfassembled monolayers within mesoporous ceramics using supercritical carbon dioxide $\left(\mathrm{SCCO}_{2}\right)$ as the reaction medium. This new methodology tremendously accelerates monolayer deposition, reduces defect density within the monolayer, increases monolayer stability, and eliminates the drying phase of the SAMMS synthesis as well as virtually eliminating the waste stream resulting

(a) Ac-Phos = 3-(2-diethylphosphatoacetamido) propyltriethoxylsilane. Prop-Phos= 3-(3diethylphosphatopropionamido) propyltriethoxylsilane. 
from the synthesis. We have now successfully demonstrated with supercritical nitrogen $\left(\mathrm{SCN}_{2}\right)$, which is also an effective reaction medium for SAMMS synthesis. $\mathrm{SCCO}_{2}$ is not entirely inert and can undergo reaction with certain classes of molecules. $\mathrm{SCN}_{2}$ doesn't suffer from this limitation and seems to be every bit as good as $\mathrm{SCCO}_{2}$ for this chemistry.

Last year, our SCF deposition studies focused on the deposition of mercaptopropyltrimethoxysilane (MPS) as our benchmark model system. Now that we have refined and optimized the synthetic protocol, we extended this capability to include the deposition of more complex materials like ethylenediamine (EDA)-silane and the phosphonate esters Ac-Phos and Prop-Phos.

\section{Lanthanide Model Studies}

Due to the expense of performing experiments with actinides, preliminary screening studies were performed using lanthanides as actinide mimics. These pre-screening studies confirmed that SAMMS can bind these target species with high affinity $\left(K_{d}\right.$ 's over 100,000 were measured, and they were commonly over 30,000), and that the binding affinities of these nanostructured hybrid materials are $\mathrm{pH}$ dependent (dropping off with increasing acidity). The binding kinetics of SAMMS was probed and found to be quite rapid - in all cases; equilibrium was reached in less than a minute!

Competition experiments were carried out and revealed only a modest effect from added iron (III) in one case (to form a six-membered ring chelate), and none at all with glycinylurea SAMMS (which forms a seven-membered ring chelate). Regeneration of SAMMS was found to be easily accomplished by a simple acid wash, affording greater than $98 \%$ of the original activity.

\section{Actinide Studies}

Promising candidates of SAMMS had their binding affinities evaluated with $\mathrm{Th}, \mathrm{U}, \mathrm{Np}, \mathrm{Pu}$ and Am. It was found that the phosphonate SAMMS had very high affinities for $\mathrm{U}, \mathrm{Pu}$ and Am. Particularly noteworthy is the high, and surprisingly $\mathrm{pH}$ independent, binding affinity of the phosphonate ester SAMMS (both Ac-Phos and Prop-Phos) for Pu. Clearly, these SAMMS are capable of playing a valuable role in the DOE cleanup effort.

Also noteworthy is that Prop-Phos SAMMS demonstrates a high affinity for $\mathrm{Pu}$ and virtually none for Am at the lower pHs. This observation suggests a very simple and direct separation of $\mathrm{Pu}$ and Am using SAMMS. In most of the experiments performed, more than $98 \%$ of the $\mathrm{Pu}$ was sequestered in the SAMMS phase, and $>99 \%$ of the Am was left behind in solution. Separation of $\mathrm{Pu}$ from Am at low concentrations is a critical need in determining the final disposition of HLW, and Prop-Phos SAMMS provides a simple, powerful, and unprecedented capability for achieving this separation. The kinetics of Pu sorption was found to only take a few minutes, and in fact is faster than typical actinide sorbents in use today. 


\section{Pertechnetate Separation}

We have also developed SAMMS to sequester tetrahedral oxoanions. Thus, we have constructed a version of SAMMS in which the pores are lined with cationic copper (II) ethylenediamine complexes, which serve as anion exchange sites that are selective for certain tetrahedral oxoanions. Cu-EDA-SAMMS was found to be moderately effective at removing pertechnetate from contaminated Hanford groundwater. Drs. Kemner and Kelley (ANL) provided detailed characterization of $\mathrm{Cu}$-EDA-SAMMS adducts, providing key insights into the binding between the copper center and the oxometallate anion.

\section{Planned Activities}

In the last year of the project, we intend to extend our actinide studies to include all of the actinides from Th through Am; evaluate the HOPO ligands in a SAMMS matrix; look at actinide competition studies, kinetics, waste simulants and ultimately possibly even actual waste samples (from tank farms, PFP or other Hanford facilities); and perform EXAFS characterization of the pertechnetate/SAMMS adduct.

\section{Information Access}

\section{Web pages}

http://www.pnl.gov/etd/product/samms/index.htm

http://www.acs.org/government/success/index.html

\section{Publications}

Feng X, L Rao, TR Mohs, J Xu, Y Xia, GE Fryxell, J Liu, and KN Raymond. 1999. "Selfassembled monolayers on mesoporous silica, a super sponge for actinides." Ceramic Transactions Vol. 93, Environmental Issues and Waste Management Technologies in the Ceramic and Nuclear Industries (IV), pp. 35-42, JC Marra and GT Chandler, eds.

Fryxell GE, J Liu, and S Mattigod. 1999. "Environmental applications of self-assembled monolayers on mesoporous supports (SAMMS)." Materials Technology 14:188-191.

Fryxell GE, J Liu, M Gong, TA Hauser, Z Nie, RT Hallen, M Qian, and KF Ferris. 1999. "Design and synthesis of selective mesoporous anion traps." Chemistry of Materials $11: 2148-2154$.

Fryxell GE and J Liu. 2000. "Designing surface chemistry in mesoporous silica." Adsorption at Silica Surfaces, E Papirer, ed. Marcel Dekker, pp. 665-688. 
Fryxell GE, J Liu, SV Mattigod, LQ Wang, M Gong, TA Hauser, Y Lin, KF Ferris, and X Feng. 2000. "Environmental applications of interfacially modified mesoporous ceramics." Ceramics Transactions, Volume 94, Environmental Issues and Waste Management Technologies in the Ceramic and Nuclear Industries (in press).

Kemner KM, X Feng, J Liu, GE Fryxell, L-Q Wang, AY Kim, M Gong, and S Mattigod. 1999. "Investigation of the local chemical interactions between $\mathrm{Hg}$ and self-assembled monolayers on mesoporous supports.” J. Synchotron Rad. 6:633-635.

Liu J, GE Fryxell, M Qian, L-Q Wang, and Y Wang. 2000. "Interfacial chemistry in selfassembled nanoscale materials with structural ordering." Invited contribution to Pure and Applied Chemistry 72:269-279.

Liu J, GE Fryxell, S Mattigod, TS Zemanian, Y Shin, and L-Q Wang. 2000. "Synthesis and applications of functionalized nanoporous materials for specific adsorption." Studies in Surface Science and Catalysis, A Sayari et al., eds. Elsevier, Vol. 129, pp. 729-738.

Mattigod S, GE Fryxell, X Feng, and J Liu. 1999. "Self-assembled monolayers on mesoporous supports for metal separation." Metal Separation Technologies Beyond 2000: Integrating Novel Chemistry with Processing, KC Liddell and DJ Chaiko, eds. The Minerals, Metals and Materials Society, pp. 71-79.

Mattigod S, X Feng, GE Fryxell, J Liu, and M Gong. 1999. "Separation of complexed mercury from aqueous wastes using self-assembled mercaptan on mesoporous silica." Separation Science and Technology 34:2329-2345.

\section{Awards}

Ken M. Kemner received the "Presidential Early Career Award for Scientists and Engineers," awarded by the President of the United States, and the "Office of Science Early Career Scientist Award," awarded by the United States Department of Energy. 